\title{
SYSTEMS AND CAUSAL LOOP THINKING IN MEDICINE: FROM HEALTHCARE DELIVERY TO HEALTHCARE POLICY MAKING
}

\author{
Author(s) / Auteur(s) : \\ Charalampos THEOCHAROPOULOS \\ Undergraduate Student \\ Faculty of Medicine, National and Kapodistrian University of Athens \\ smd1600219@uoa.gr
}

\begin{abstract}
Résumé :
The human body is regarded as a system of high complexity, not only because it is consisted of millions of interrelated and interdependent functional units -the cells-, but because it is also an evolving system. It changes over time, initially to achieve the full growth of organs and bones but subsequently as a response to environmental factors to retain its vital internal indexes stable, to achieve homeostasis. In this context, the in depth understanding of the connections between these indexes that drive the dynamics of the system is crucial. Yet, malfunctions occur and their accumulation causes diseases, which are regarded as internal crises that due to tight relations between the different organ systems, affect various body parts. The application of systems and causal loop thinking while combating diseases is examined and the need to treat not the body part that is ailing, but the patient as a system is underscored through examples of diseases. The importance of examining the risk and trigger factors of diseases from a systemic perspective is also highlighted through examples from the medical literature. The patient itself is viewed in the context of the Swiss Cheese Model and the causal agents that lead to a system failure and patient harm are examined, as well as ways of strengthening the healthcare system in order to minimize the vulnerabilities and the possibility of failures, with particular regard at modelling doctor-patient relations as Paskian Conversations. The Triumvirate of Public Health concept is discussed as a valuable practice in the healthcare policy making sector, regarding both top-down and bottom-up modes.
\end{abstract}

\section{Keywords / Mots-clés :}

causal loop thinking, homeostasis, dynamic equilibrium, public health, healthcare policy making, swiss cheese model, conversation theory, medical cybernetics

\section{INTRODUCTION}

Heisenberg has quoted that "It is probably true quite generally that in the history of human thinking the most fruitful developments frequently take place at those points where two different lines of thought meet". In this article we will explore the meeting point of medical science, health care and systems paradigm. If we were to trace the relationship between the above disciplines, we might have to go as back as ancient times when Hippocrates stated that the body cures itself, paving the road towards what we it is referred today as homeostasis, self-regulation and feedback, which are important ingredients of systems thinking. Biological and medical sciences in fact contributed to the ignition of systems science and cybernetics. Pioneers like von Bertallanfy, who is attributed the father of systems thinking, and Ross Ashby, a major figure in cybernetics, prove Gordon Pask' remark that "perhaps the earliest cybernetic thinking comes within the compass of physiology" (Pask, 1961). On the other hand, it seems that the systemic paradigm is not a pronounced paradigm in health care as opposed to the reductionist paradigm. The advance in medical knowledge, tightly related with the advance in computer power, is of a continuingly increasing ratio and results in exponential growth of the Paskian "entailment mesh". Epistemologicaly, the systemic paradigm acts as a complexity compensator by providing leverage points and archetypes. In this context, we will explore facets of systems thinking that can serve as tools for the systemic doctor, namely CLDs, the Swiss Cheese Model and Paskian learning conversations. 


\section{FACET 1:}

\section{MAPPING MEDICAL PROCESSES WITH CAUSAL LOOP DIAGRAMMS}

The human organism is the result of about 3 billion years of evolution crafted and catalyzed by natural selection in a way that mechanisms shared by the reproductively successful agents have been gradually chiseled and optimized to maximize the possibility of survival and reproduction. Thus, if seen as an entity, the body's utter aim is to preserve the conditions necessary for the life of the elements. This is achieved by ensuring that the vital indexes driving its dynamics are kept within some tight boundaries so that the body can establish itself within the zone of vitality. Thus, the quintessence of the body's functionality lies in the preservation of its own existence. However, being an open system, it is in constant exchange of both energy and mass with its environment resulting in an overflow of both internal and external stimuli that can be potential disequilibrium catalysts of the vital index values which ensure the body's operation. Reacting to this potentially catastrophic flood of stimuli the body has developed a stability mechanism which is known as homeostasis control. Therefore, as described by Cannon (1932), for that constancy to be achieved, any tendency towards differentiation simultaneously meets regulatory mechanisms resisting the change, whose conjoint action, each aimed at maintaining a certain factor, constructs a homeostatic self-regulation system. The main way through which that assembly functions is reflective and feedback systems that operate in balancing loops. Such circuits are mainly consisted of sensors that detect changes in the monitored variable's values and generate impulses, a process defined by Magendie as a reflex, carried through afferent pathways towards an assembly, dedicated in what McCulloch describes as redundancy of computation. Hence, it amalgamates such messages and produces signals which, through efferent pathways, galvanize effectors towards minimizing the initial disturbance and restricting it in the viability compatible levels. That arbiter receiving sensor's information and responses is the brain, a subsystem of the organism, acting, from a cybernetics point of view, as a controller and more specifically as an adaptive controller, given that it seeks dynamic equilibrium within certain range rather than any kind of stability.

For these balancing and regulative process to be more easily understood, the modelling through Causal Loop Diagrams (CLDs) is examined as CLDs describe both dynamic equilibria and disequilibria and one can find both in the human body. Through the representations of basic processes modelled using Vensim, the robustness of CLD diagrammatic language in effectively describing such processes and simplifying their underlying dynamics is illustrated. Moreover, the very important feature of the human organism, its actual function as a control device, in which numerous decision makers may be engaged, redundancy of potential command according to McCulloch, becomes profound. Furthermore, CLDs can be mathematically simulated if one requires numerical output. Stock and Flow diagrams is another way to conceptualize such dynamic processes. Yet, they require a set of differential equations to govern these processes and they are more mathematically intense than CLDs.

As an example of CLD modelling of a balancing loop, the regulation of Mean Arterial Pressure (MAP) is chosen. MAP is defined as the average blood pressure in the arterial circulatory system in each cardiac circle. It is a significant hemodynamic factor ensuring adequate prefusion and blood flow to the organs and therefore adequate cellular oxygenation in line with metabolic rates and oxygen demand. Being the arithmetic product of Cardiac Output (CO) -the volume of blood pumped by the left ventricle per unit time- and the Total Peripheral Resistance (TPR) -the sum of resistances to blood flow offered by all systemic vasculature-, it is that changes in any of the two result in changes in the MAP. Thus, determinants of the $\mathrm{CO}$ and the TPR serve as determinants for the MAP. More specifically, arterial baroreceptors -the carotid sinus baroreceptors and the aortic arch baroreceptormonitor pressure levels through nerve endings sensitive to stretch or distortion and generate impulses. These impulses are carried through afferent neurons towards the medullary cardiovascular control center, with a certain rate proportional to the MAP. However, if a disturbance in the level of MAP occurs the rate of neurons' discharge is altered similarly to the causing discrepancy; lower MAP leads to a decreased rate of discharge, whilst higher MAP leads to an increased rate. The differentiation of travelling impulses, processed by the medullary cardiovascular control center, results in alteration of the sympathetic and parasympathetic nerves' activity in a way that depends on the kind of the initial 
disturbance. If the stimulus is lower MAP then the parasympathetic activity on the heart rate is reduced, resulting to a higher HR, while sympathetic nerves function towards the re-establishment of MAP within normal levels through 4 ways: increase of venous pressure, increase of stroke volume, increase of HR, increase of Total Peripheral Resistance through reduction of the arterial radius. If the stimulus is higher MAP, the above-mentioned operations are reversed. Except for the main feedback loop of baroreceptors, the MAP is regulated through hormonal and local controls, as shown in figure's 1 model, signifying the complexity of regulation mechanisms for a single variable. Therefore, CLD modelling of such a plethora of homeostatic loops, could act as complexity compensator for both the student and the young doctor, while the combination of multiple CLDs could highlight potential links between ostensibly unrelated variables, trigger causes and risk factors for diseases. It must be underlined, however, that causal loop thinking is eventually developed by practiced physicians. Through the construction of a concrete statistic base and the long interaction with the human body, experienced doctors are able to quickly simulate loops given the symptoms. Thus, CLDs become automated, with the symptom being rapidly linked to its underlying cause without the deliberate procession of interim steps.

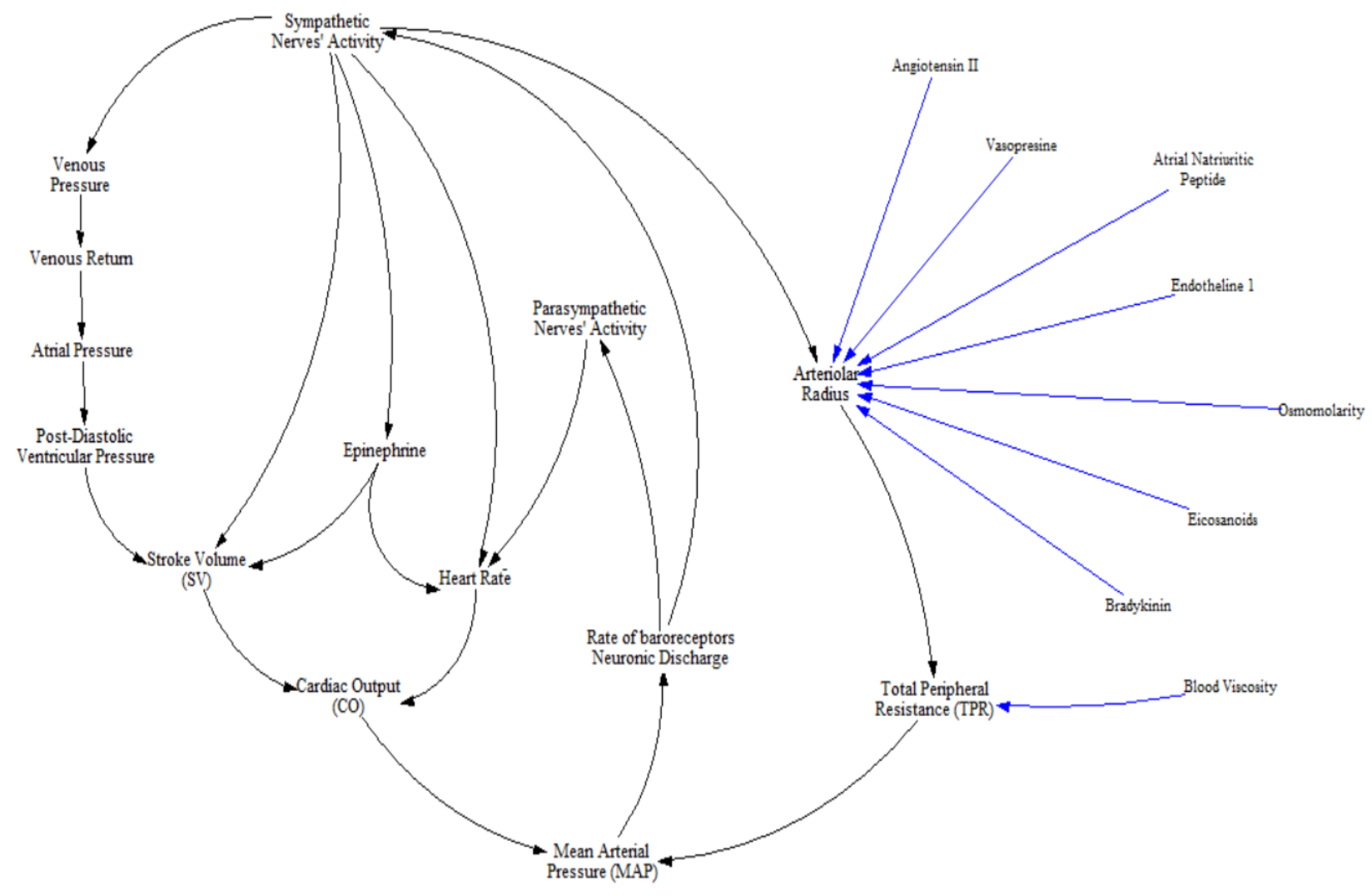

Figure 1: Causal Loop Diagram of the regulation of MAP as described in the text. Blue arrows indicate local and hormonal controls that are part of other loops. 


\section{FACET 2:}

\section{THE SWISS CHEESE MODEL,}

\section{A SYSTEMIC APPROACH TO MEDICAL FAILURE}

The shift to the $20^{\text {th }}$ century was marked by the consensus view of the medical community that "The health care system isn't as safe as it could be", popularized through landmark reports such as "To err is human", by the Institute of Medicine (Kohn, Corrigan \& Donaldson, 2000) and "An organization with a memory" (Donaldson, 2002). Both reports were devoted to breaking the silence and inaction surrounding medical errors and preventable harm, with the first one stating that 44,000 to 96,000 people died at that time in the U.S. in a yearly period due to medical errors and the latest estimating the number of adverse events to 850,000 a year in the U.K. Almost 20 years after these seminal publications, considering the increased rate of publications and the augmented general interest regarding all types of patient safety issues stimulated by these reports (Stelfox, Palmisani, Scurlock, Orav \& Bates, 2006), one could logically expect that much progress towards the fulfillment of the set goals, the materialization of the recommendations and therefore the reduce of patient harm would have been achieved. However, the recently published study of the Johns Hopkins University which made an estimation that up to 250,000-400,000 deaths a year are caused by medical errors (Makary, Daniel, 2016), came to confirm through incontrovertible evidence what was tactfully noted by papers and publications during the last 5 years (Leape, Berwick, 2005): There is still much to be done towards converting the Health Care System into a High Reliability Organization (HRO).

Although the health care system shares almost all the characteristics of an HRO, it lacks the ability to function in low error rate which is the signature status of HROs. In order to understand the causes of that inability, we have to define the purpose of the health system and who and in which way is involved towards achieving that purpose. A health system can be defined as "The combination of resources, organization, financing, management that culminate in the delivery of health services". Therefore, as in all systems there are agents functioning and interacting towards the promotion, restoration and maintenance of health and of the general well-being. In the organizational spectrum of Healthcare, three primary actors can be identified: i) Government, ii) Hospitals, iii) Medical Personnel. We explore these actors in the context of the Swiss Cheese Model. Hospitals and medical personnel can be examined as sub-categories of a more general category labelled "Providers". Also, healthcare services can be delivered by individual doctors not operating in hospitals and therefore the defensive layer "Clinics" is absent in these cases. We don't aim at an in-depth analysis of flaws and their causes, rather we focus on specific examples illustrating how systems thinking could assist the exegesis and prevention of patient harm occurrence. All layers that we described can be divided in sub-layers, for the concept of latent conditions and active failures to be better understood.

The "Swiss Cheese Model" of accident causation, proposed by James Reason, serves as a systemfocused approach to error and it is widely used since its initiation in a variety of high-risk fields. The SCM portrays the multiple defensive layers of an organization against harm as cheese slices. In these layers there are holes, analogous to breakdowns in these safeguards, which if aligned lead to hazard trajectory and accidents. It, therefore, highlights that in complex systems, for a catastrophe to happen, multiple successive failures must appear to the different barriers, as it is their concatenation and conjoint action that provokes harm. These failures are categorized into latent conditions, meaning dormant pathogenies of the given organization, and active failures, meaning the trigger causal factors. In this manner, it is possible to shift focus from the sharp-end, the individual health-care professionals, to the system as a unity and its error-producing conditions. Thus, two major facets emerge: The first is that harm can be prevented by any of the individual defenders, even if the rest layers malfunction. The second is that by identifying and tackling latent conditions in the "blunt end", potential active failures cannot ignite the causal chain. It is also important to mentione that even though the SCM is perceived as an easily used means of communicating information between investigators during risk assessment and management, there is evidence that the understanding and interpretation of specific features of the SCM vary among quality and safety professionals (Perneger, 2005). One can therefore argue that the misconception during its usage, and of other similar models, may halt its advantages and produce adverse effects in the sense of mis-including a causal factor as tackled. Therefore, the ill-formed 
understanding of such tools can be regarded in the context of the SCM as a latent error fertilizing the ground for accidents to happen.

\section{Governance}

The first layer we explore is that of the government. In that layer holes are mostly latent conditions. In this context we could identify policy-making and expenditure in health per capita as potential holes. As for policy making two major categories exist: top-down and bottom-up policy making. In top-down policy making, as in all complex systems, interaction of three major actors is essential, namely technocrats, local officials and civil leaders, in an approach described as the "Triumvirate of Public Health". Technocrats, in this case, is the group of people that are well-versed when it comes to matters of epidemiology, public health and medicine and are cognizant of developments in the field. Local officials, as local implementers, can ensure the sustainable application of the policies in a way understandable by the public and civil leaders have the power to imply laws. These three should be engaged in a stimulus-response circle, continuously interacting through a shared and mutually understandable language, that can be built, as described in facet 3 for a robust and applicable policy to be drawn. In bottom-up policy making, discretion by agents is the underlying premise, where the expertise of people is utilized to increase the probability of success. One important thing to be particularly noted about healthcare is that healthcare providers are not always professionals, and to a great deal healthcare services are provided by non-medical population, especially in emergency cases. Therefore, combining these facts, we could argue that from a systemic perspective Top-down policymaking could be used to achieve a paradigm shift towards Bottom-up policy making, by engaging the general population through constructing medical knowledge curriculum. Another latent condition is a low expenditure in health care per capita, or inefficient usage of the expenditure. In the first case people are forced to pay more in healthcare services and impoverishment occurs, leading to further deterioration in their health status (Verguet, Memirie \& Norheim, 2016 ; Liu, Rao \& Hsiao, 2003). In the second case capital spent in the healthcare domain fails to create profound results.

\section{Civic Leader}

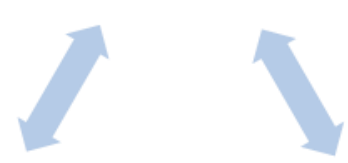

\section{Technocrat}

Figure 2: Interaction of the major actors interacting in healthcare policy making as a closed stimulus response circle.

\section{Hospitals and clinics}

As the second layer, we examine healthcare in the level of hospitals and clinics. If seen from a systemic and cybernetic point of view, clinics are entities consisted of numerous interconnected parts, namely doctors, nurses and other healthcare professionals, that co-operate towards improving the health status of their system's main input, the patients. Considering the entity of a hospital as a safeguard, we focus on regulation, managerial and organizational issues which increase harm occurring possibilities. We explore the role of individual healthcare professionals as a separate slice. In this layer, one can find both latent conditions and active failures. Some examples of latent conditions are the following: 
A) Non-existing or ineffective monitoring of supply usage

B) Lack of computerization of processes such as prescription of drugs

C) Lack of effective work allocation

D) Inability to attract resources

Such conditions can contribute to many hazard trajectories as they allow active failures to happen. An example of that is drug shortages. The impact of drug shortages is multidimensional, with over $50 \%$ of health care practitioners believing that shortages have influenced practice and caused inferior patient care (Caulder, Mehta, Bookstaver, Sims, Stevenson, 2015). Drug shortages cause 1\% to 5\% error rates in hospitals and $60 \%$ of the time drug shortages create unsafe conditions for patients and staff (Baumer, Clark, Witmer et al., 2004) serving as causal factors for medical errors. From the abovementioned latent conditions, the concept of rejection of resources to unsuccessful and inefficient agents is no strange to the human body itself. More specifically, in the pulmonary system the adaptive effect generated by decreased P02 in pulmonary vessels surrounding an alveolus, indicating decreased efficiency of the alveolus to supply $\mathrm{O} 2$, is vasoconstriction. In this way less blood reaches such dysfunctional alveoli and more is available for well-operating ones.

\section{Medical Personnel}

As the last safeguard and the one with the closest and most direct interaction with patients and general population we explore physicians and the rest of paramedical personnel. According to the IOM errors can be categorized as i) Diagnostic, ii) Preventive, iii) Treatment, iv) Other. We focus on the most notable kind of errors that could be approached and tackled from a systemic perspective.

\section{Prescribing errors}

To begin with, prescribing errors, included in the "Treatment" type of errors, account for one of the most common sharp-end mishaps that lead to preventable harm. An analysis of 24 studies calculated the range of error rates to 2-514 per 1000 items prescribed and 4,2-82\% of patients or charts reviewed, without, however, reaching clear meaningful conclusions about the root causes, yet highlighting the need for future research on the field (Ross, Bond, Rothnie, Thomas, Macleod, 2009). CLD modelling could function towards the optimization of the physician's working knowledge, illuminating the links between seemingly non-directly related body parts, in the context of drug impact. For example, Aspirin, a widely used nonsteroidal anti-inflammatory drug, acts as an acetylating agent irreversibly inactivating the prostaglandin forming cyclooxygenase (COX). Two main isoforms of COX are COX2 that generates pro-inflammatory mediators and COX-1 that generates prostaglandins vital for the preservation of essential functions, such as the maintenance of the gastric mucus layer and platelet aggregation. It is, therefore, clear that the ignorance of the CLDs in which, in that case, aspirin intervenes may lead to undesirable effects. For example, in patients with a history of gastrointestinal problems, aspirin can cause: promotion of peptic degradation, decrease of viscosity, and reduce of the ability to resist hydrogen ion penetration, all leading to susceptibility to severe upper gastrointestinal complication (Rodríguez, Hernández-Díaz \& de Abajo, 2001). Prescribing errors could also be the result of an inefficient communication between the physician and the patient, that also affects the diagnostic process and patient's satisfaction as discussed in the following paragraphs. 


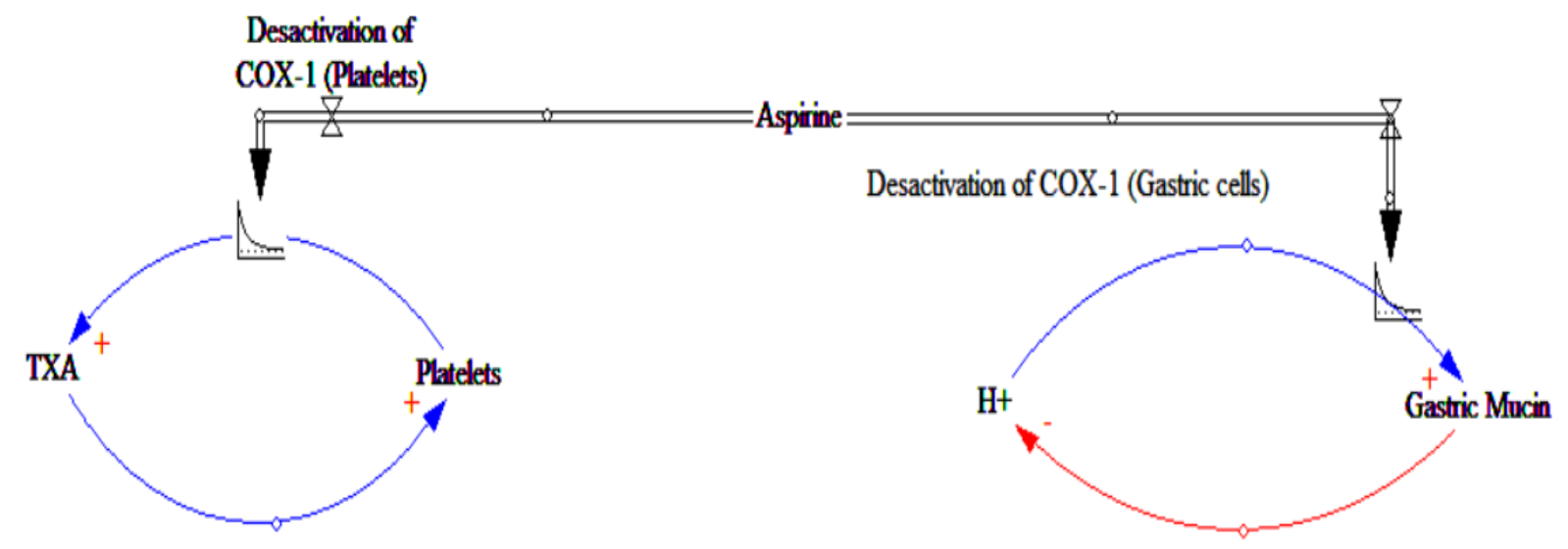

Figure 3: The impact of aspirin as a COX 1 inhibitor in two different cell populations. Aspirin can be prescribed as an anticoagulant due to its effect on Platelets' COX 1, still it can cause undesirable effects to certain patients due to its similar effect on COX 1 present in gastric endothelial cells, as explained above. The depicted loops have been simplified with only the major variables being included.

\section{Misdiagnosis}

Diagnostic errors form another reason contributing to the deprivation of healthcare quality and patient harm and are a common flaw in the clinician's layer. An analysis of three studies yielded a rate of diagnostic error of $5.08 \%$ a percentage equal to 12 million US adults every year, with the researchers estimating half of these error as potentially harmful (Singh, Meyer \& Thomas, 2014). The causes of diagnostic errors are multiple, including the utilization of outmoded tests, the wrong interpretation of test results and many more which become possible due to holes in the pre-existing layers, as shown in figure. We particularly focus on an example that illustrates the need for a systemic approach by the doctor. The complexity of the human body can give rise to issues that require the enhancement of the reductionist model in favor of a more systemic one that takes into consideration the self-regulation processes described in facet 1 . In this context, disequilibrium as seen by change of a vital index can by either seen as a malfunction that requires immediate treatment or as an intended disequilibrium that is part of a self-healing process. The doctor that is not able to realize the systemic nature of that disequilibrium might disrupt the self-curative process, leading to deprivation of patient's health. A characteristic example is the fall in the plasma Fe (iron) concentration that occurs in several infections. That decrease is, in fact, an adaptive defensive effect of the organism aimed at depriving the infectious agents of the Fe that is essential for their replication. However, if this respond of the organism with inflammatory cytokine-induced hypoferremia is perceived as a symptom caused by the infectious agent and treated with iron supplements, the prognosis of the disease gets worse.

\section{Ineffective Doctor-Patient Communication}

Another hole in the medical personnel's layer that still occurs with a worrisome ratio is the failure of healthcare professionals to establish an effective communication with patients. As already stated, such an inability hinders both the process of diagnosis and the creation of a treatment plan, but also leads to patient dissatisfaction. Patient dissatisfaction, despite the seemingly long-achieved adoption of Engel's Biopsychological Model (Engel, 1977), was often treated as a minor flaw in the healthcare delivery, especially if co-existing with a satisfactory health status of the individual. However, numerous studies have shown that patient satisfaction is related to improved guideline adherence, more effective application of treatment plans and in the long run lower mortality levels and better quality of life (Lee, Moriarty, Borgstrom, Horwitz, 2010 ; Haskard Zolnierek, DiMatteo, 2009 ; Ratanawongsa, Karter, Parker, et al., 2013 ; Wong, Fielding, 2009). Such results lead to a change towards the increased use of patient satisfaction survey tools for evaluating the overall performance of the healthcare system (AlAbri \& Al-Balushi, 2014). Still, disrespect and ineffective communication combine for $13.43 \%$ of the total reasons of patient dissatisfaction (Lee, Moriarty, Borgstrom \& Horwitz, 2010), while the inability 
to provide sufficient information in an explanatory and understandable way to the patient acts as a potential hindrance for the intended therapeutic synergy. That is, for example, of particular importance in the context of the anti-vaccination movements where vaccine sceptics should be met with concrete information by well-trained and communicative physicians. However, taking into consideration the long-set and still unfulfilled aim to engage medical students to psychology and Behavioral and social sciences (B\&SS) (Pickren, 2007), we propose the integration of Conversation Theory in the course of not only doctor-patient relation, but doctor's relation with the rest of the front-line agents operating towards the defined mutual goal.

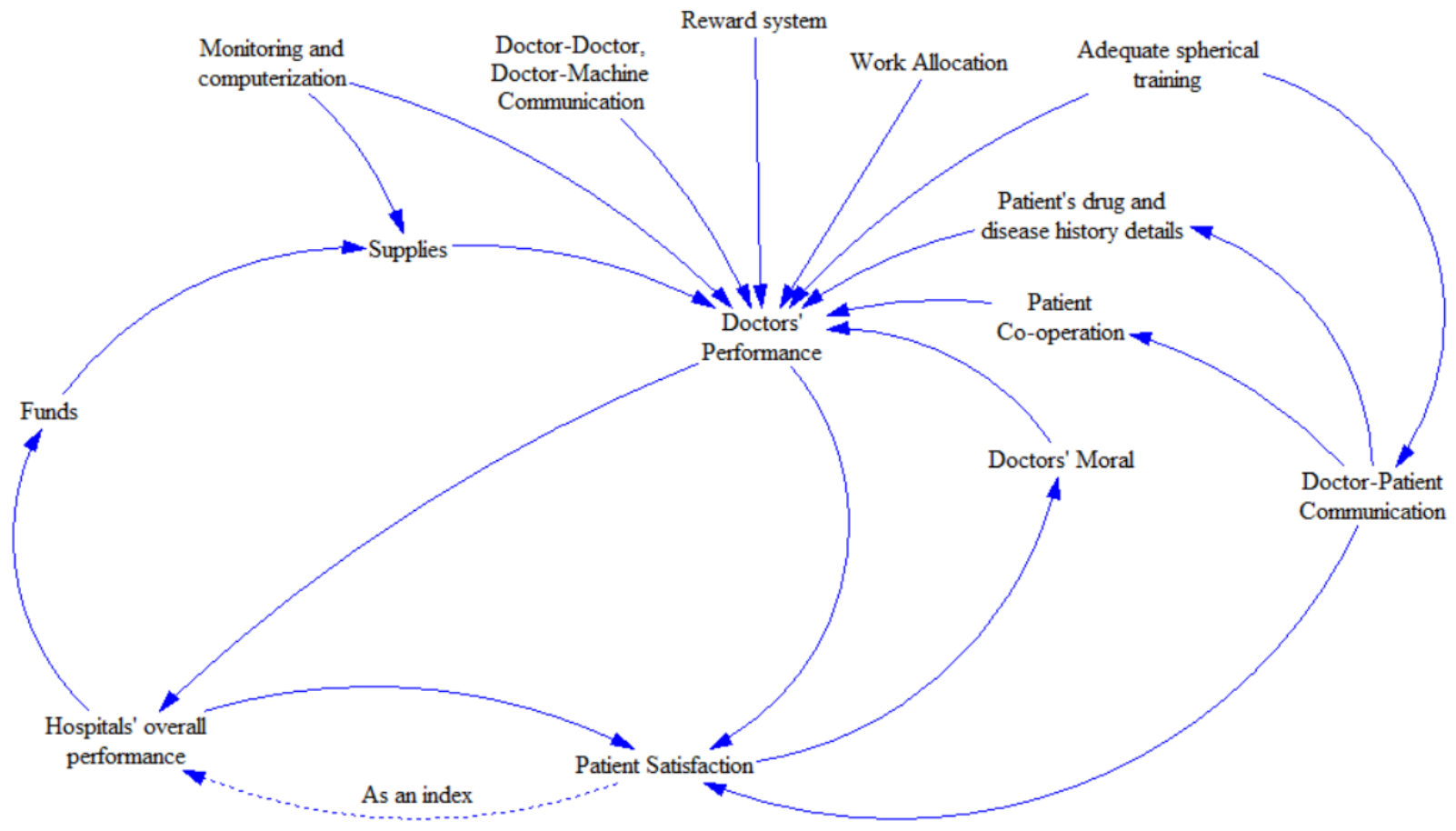

Figure 4: Causal Loop Diagram of doctors' performance.

\section{FACET}

3:

\section{MEDICAL LEARNING CONVERSATIONS}

Conversation Theory (CT) was developed by Gordon Pask. The Paskian conversation is a dialog that externalizes internal processes within a contractual and normative framework. The participating agents negotiate the normative framework of the conversation in a natural language $\mathrm{L}^{*}$ but the conversation itself is carried in a conversational language L. According to Pask, a conversation involves a topic $\mathrm{T}$ and is actually a model building operation implemented in a cognitive reflection device. The whole process concludes in a mutual agreed stable entailment mesh or ontology that can be stored in a memory device. In this manner, Pask creates a cybernetic framework of learning that as every cybernetic model, can be used for human-human, human-machine and machinemachine interactions invariably. We propose that Pask's Conversation theory can be applied in doctor patient-relation, doctor-doctor relation and doctor-machine relation. 


\section{Doctor-Patient Conversation}

The relation between a physician and a patient is of vital importance as it is a key element for a therapeutic synergy to be established. A well-organized learning conversation can enhance diagnosis, treatment plan and treatment application. During the learning conversation both the doctor and the patient function as learning agents committed to the basic conversation rules.

a) A natural language $L^{*}$ must be decided between patient and doctor

b) A conversational language must be decided on or created. This language can involve words symbols and mathematical elements and notations

c) A system for cognitive reflection has to be decided

d) A model building process and a shared model must be concluded

Usually, doctor patient interactions are seen in an operative manner according to which the doctor acts on the patient by intervening in the patient's lifestyle. In the cybernetic perspective that the patient doctor system is a learning system and must engage in a structured learning conversation in which both doctor and patient create their common entailment mesh of the topic. Assuming that the natural language is the same and easy agreed upon, it is up to the doctor to propose a conversational language $\mathrm{L}$ so the conversation can commence. If $\mathrm{L}$ is too technical then cognitive reflection from the part of the patient might be difficult. If $\mathrm{L}$ is close or equal to the $\mathrm{L}^{*}$, then the cognitive reflection of the patient might not be accurate. Doctors should commit to ensure the productive engagement of the patient in the learning conversation and his sufficient familiarization with language L, through scaffolding and negotiation of meaning. The success of that doctor-patient conversation in the broader context of diagnosis and therapy, could be signaled by the ability of the patient to "reconstruct the concept of Topic T". The doctor patient conversation can play an important role in identifying all the elements that comprise the deviation from the norm of the patient vital indexes which is the crucial part in diagnosis. Systemics offer a conversational language Ls (Systemic Language) as used in the Paskian notation of a learning conversation. This language consists of concepts and notations that can be found in the systems thinking field. Ls can enhance the doctor -patient conversation but can equally well serve the medical epistemological conversation between communities of interest. Learning conversations can transform heuristics to archetypes as well as transform tacit knowledge to sharable models. Learning conversations can also help identify fragmented and uncomplete ontologies. Applying the principles of Conversation Theory also help interchange of knowledge between subjects in the form of archetypes and persistent structures. Applying the principles of Conversation Theory and the establishment of an L and the process towards it, can also help the doctor understand the purpose of the patient

\section{Doctor-Doctor Conversation}

CT in the context of knowledge reflection between physicians, can create a mechanism for transforming implicit knowledge to explicit. It is well known that doctors as practitioners derive heuristics that they utilize in a conscious or unconscious level. This valuable working knowledge can be transformed to formal knowledge through the conversation mechanics, as described above, of Pask's conversation theory. Individual doctors or communities of doctors can engage in a structured conversation in order to create their ontology or entailment mesh. Although, knowledge interactions are the most prominent interactions between practitioners, the use of a systemic methodology can ensure that the product of this interaction is a mutually agreed knowledge base that can be constantly updated and enhanced. Furthermore, as stated, the rapid advance in computational power results in an equally paced development in the medical knowledge, the techniques and equipment available, that seems to surpass the ability of health professionals to interpret it. For that issue to be tackled, new specializations emerge that focus on the in depth understanding of fractions of the total medical knowledge. In this context, CT can assist the normalization of the knowledge background of different doctors, who may not share the same L. 


\section{Doctor-Machine Conversation}

The creation of a learning and thinking cybernetic agent in the medical sector is an ongoing evolutionary process that has undergone many phases such as medical databases, medical expert systems, medical intelligent agents, data mining, big data, artificial medical intelligence, machine learning and deep learning. Envisioned by Gordon Pask, this cybernetic agent will be capable to engage in learning interactions with the human agent through the construction of an ontological object language and a cognitive reflection interface. The conversation between the human agent and the machine agent through Pask's "teachback" effect can lead both serialist and holistic learners to an efficient exploration of serial processes and higher order relations. Today, this medical practitioner machine conversation can take the form of querying an AI machine for insights and meaningful statistical representation. Learning conversations with the AI agent can help the practitioner update his field knowledge, minimize human error and cope with thinking and decision-making biases. Technology is constantly enhancing the objective language L used for the artificial - human agent communication. With the computational power sharply rising through parallel computing, supercomputing, cloud computing and quantum computing, $\mathrm{L}$ increasingly resembles natural language in description and communication power. Cloud technologies can make these learning cybernetic agents accessible through all available communication means, dedicated terminals, PCs smartphones, VR sets etc.

\section{CONCLUSION}

The medical practitioner operates in a complex environment mainly due to the inherent complexity of the human body. In his quest to match complexity to an operational level, complexity compensation strategies are applied: protocols, heuristics, hierarchies, best practices, statistical estimations etc. Most of these complexity compensation tools are of reductionist nature as they have been proven fast and agile, able to deal with urgency, a crucial factor that augments the complexity of the medical environment. From the scope of Professional Systemics, we argue that the complexity compensation toolbox of the medical practitioner can be enhanced with some purely systemic tools such as Causal Loop Diagramming and Stock and Flow mapping, agent-based system dynamics and discrete simulation, game theory, Conversation Theory learning cybernetics, systemic strategic models such as the Viable System Model or Swiss Cheese model and multimethodology dialogue tools. CLDs and SF mapping can reveal hidden unpredicted and sometimes not profound causal loops that govern human body processes. This can help a practitioner, for example, to translate crucial index deviations either as illnesses that need interventions or as local disturbances in a self- healing process that might not need to be interrupted at that phase. CLD and SF mapping can contribute to enhance ontologies that more efficiently capture the relationship between root cause and symptoms. The Swiss Cheese model is an excellent tool to model the inherent risk found in any complexity compensation effort. The selection of the intervening layers helps a practitioner to balance efficiency with risk so as to work at the best possible ratio. Unneeded layers can be identified, crucial layers can be modified and missing layers can be added in a static or dynamic way. This model is highly systemic as it is recursive in all the organizational layers in medical practice, from the sole doctor to national health systems. Finally, Conversation Theory proposes a cybernetic formation to one of the most important social process, that of learning. Cybernetics' inherent ability to treat human and machine with the same agent-based operational models provides Conversation Theory with the ability to create controllers for the multidimensional learning conversations of a medical practitioner, namely the ones with patients, fellow practitioners, the community of practice, the medical scientific community, the medical industry, the technology industry etc. The practitioner seeks for better, enhanced and dedicated languages and cognitive reflection platforms that can lead to mutual understanding and ontology building. CT can facilitate the transformation of tacit knowledge to explicit and organizational knowledge, a very important process since much of the working knowledge of medical practitioners is encoded in hints, heuristics and best practices. 


\section{RÉFÉRENCES}

PASK, G. (1961). An approach to cybernetics. Hutchinson \& CO, London.

CANNON, W. B. (1932) Wisdom of the Body. W.W. Norton \& Company, Inc, New York

KOHN L. T., CORRIGAN J., \& DONALDSON M. S. (2000). To err is human: Building a safer health system. National Academy Press, Washington DC.

DONALDSON L. (2002) An organization with a memory Clinical Medicine 2(5):452-7

STELFOX, H. T., PALMISANI, S., SCURLOCK, C., Orav, E. J., \& Bates, D. W. (2006). The "To Err is Human" report and the patient safety literature. Quality \& Safety in Health Care, 15(3), 174178.

MAKARY M., DANIEL M. (2016) "Medical error-the third leading cause of death in the US". BMJ 353:i2139

LEAPE LL, BERWICK DM. (2005) Five Years After To Err Is Human What Have We Learned? JAMA. 293(19):2384-2390

PERNEGER TV. (2005) "The Swiss cheese model of safety incidents: are there holes in the metaphor?" BMC Health Services Research. 5:71.

VERGUET, S., MEMIRIE, S. T., \& NORHEIM, O. F. (2016). "Assessing the burden of medical impoverishment by cause: a systematic breakdown by disease in Ethiopia". BMC Medicine, 14, 164.

LIU, Y., RAO, K., \& HSIAO, W. (2003). "Medical Expenditure and Rural Impoverishment in China". Journal of Health, Population and Nutrition, 21(3), 216-222.

CAULDER CR, MEHTA B, BOOKSTAVER PB, SIMS LD, STEVENSON B. (2015) "Impact of Drug Shortages on Health System Pharmacies in the Southeastern United States". Hospital Pharmacy. 50(4):279-286.

BAUMER AM, CLARK AM, WITMER DR, et al. (2004) "National survey of the impact of drug shortages in acute care hospitals". Am J Health Syst Pharm. 61:2015-2022.

ROSS S, BOND C, ROTHNIE H, THOMAS S, MACLEOD MJ. (2009) "What is the scale of prescribing errors committed by junior doctors? A systematic review". British Journal of Clinical Pharmacology. 67(6):629-640.

RODRÍGUEZ, L. A. G., HERNÁNDEZ-DÍAZ, S., \& de ABAJO, F. J. (2001). Association between aspirin and upper gastrointestinal complications: Systematic review of epidemiologic studies. British Journal of Clinical Pharmacology, 52(5), 563-571.

SINGH, H., MEYER, A. N. D., \& THOMAS, E. J. (2014). The frequency of diagnostic errors in outpatient care: estimations from three large observational studies involving US adult populations. BMJ Quality \& Safety, 23(9), 727-731.

ENGEL G. (1977) The need for a new medical model: a challenge for biomedicine. Science 196(4286):129-36.

LEE AV, MORIARTY JP, BORGSTROM C, HORWITZ LI. What can we learn from patient dissatisfaction? Analysis of dissatisfying events at an academic medical center. Journal of hospital medicine: an official publication of the Society of Hospital Medicine. 2010;5(9):514-520.

HASKARD ZOLNIEREK K.B., DIMATTEO M.R. (2009) "Physician Communication and Patient Adherence to Treatment: A Meta-analysis". Medical care. 47(8):826-834.

RATANAWONGSA N, KARTER AJ, PARKER MM, et al. (2013) "Communication and Medication Refill Adherence: The Diabetes Study of Northern California". JAMA Intern Med. 173(3):210-218.

WONG WS, FIELDING R. (2009) "A longitudinal analysis of patient satisfaction and subsequent quality of life in Hong Kong Chinese breast and nasopharyngeal cancer patients". Medical Care. 47:875-881.

AL-ABRI, R., \& AL-BALUSHI, A. (2014). Patient Satisfaction Survey as a Tool Towards Quality Improvement. Oman Medical Journal, 29(1), 3-7. 
LEE, A. V., MORIARTY, J. P., BORGSTROM, C., \& HORWITZ, L. I. (2010). What can we learn from patient dissatisfaction? Analysis of dissatisfying events at an academic medical center. Journal of Hospital Medicine : An Official Publication of the Society of Hospital Medicine, 5(9), 514-520.

PICKREN, W. (2007). Psychology and medical education: A historical perspective from the United States. Indian Journal of Psychiatry, 49(3), 179-181. 